en addition préalable à des graines humidifiées agit sur la matière azotée qu'il peptonise et la rend ainsi mieux utilisable pourl'alimentation.

\title{
BIBLIOGRAPHIE
}

O. Jensen. Revue générale du lait 1906.

J. Sarthou. C. R. Ac. Se. t. CL. 1910 p. 119.

J. SARthou. Journal de Pharmacie et de Chimie 1910.

M. Nicolle. Ann. Inst. Pasteur, t. XXI, 1907.

Bouska. Revue générale du lait, t. III, 1903.

Macḱ. Traité de Bactériologie, t. II, 1913.

Portier. C. R. t, A. Sc. CLXV, p. 267.

Marohoux. Ann. Inst. Pasteur, t. XVII, p. 177, loc. cit.

M. Fouassier, C. R. Ac. Sc. t. CLXX, 1920, p. 145.

M. FouAssier. $C, R$. Ac. Sc. t. CLXXI, 1920, p. 327.

M. Fovassier. C. R. Académie d'Agriculture, t. VII, 1921, p. 706.

\section{EXPÉRIENCES TECHNIQUES CONCERNANT L'INFLUENCE DE LA TEMPÉRATURE DU BARATTAGE SUR LA TENEUR EN MATIËRE GRASSE DU BABEURRE, PROVENANT DE CRÈME RÉFRIGÉRÉE A TRÈs BASSE TEMPÉRATURE.}

\author{
par W. Van DAM.
}

Par l'élévation de la température du barattage, la teneur en matière grasse du babeurre, provenant d'une crème déterminée et travaillée, pour le reste, dans les mêmes conditions, augmente. Ce fait est universellement connu, et fut constaté dans nombre d'expériences. Encore dernièrement au Danemark (1), des expériences sur très grande échelle en donnèrent confirmation : 24 expériences furent faites dans deux fabriques; il y eut trois températures différentes, dont l'écart était de $+3^{\circ} \mathrm{C}$. ; les moyennes suivantes furent établies pour la teneur en matière grasse du babeurre : Température la plus élevée : $0,877 \%$. Température intermédiaire : $0,586 \%$. Température la moins élevée : $0,490 \%$.

Pour autant qu'il m'est connu, on n'utilisa jusqu'ici dans de telles expériences techniques que de la matière grasse de crème, qui n'était pas dans des conditions d'équilibre à la suite de réfrigération à trè̀ basse température, et comme il fut constaté dans les expériences de laboratoire que dans le babeurre provenant de crème dont la matière grasse n'est pas dans des conditions d'équilibre, la teneur en matière grasse est toujours plus élevée qủe dans celui de crème où ces conditions ont été réalisées (2), il était intéressant d'examiner, à ce sujet, les

(1) 120 de Beretning fra Forsogslaboratoriet, 1926

(2) Le Lait, 1928, p. 369. 
données des expériences à la fabrique. Il s'agissait donc en premier lieu de constater si on pouvait considérer, comme une règle générale, le fait constaté dans toutes les expériences du laboratoire qu'une élévation de la température du barattage de crème réfrigérée à très basse température provoquait toujours une hausse de la teneur en matière grasse du babeurre. La crème fut traitée selon la méthode décrite dans l'article précédent (1). Après réfrigération à très basse température durant \pm 22 heures, le levain lactique fut additionné et le barattage fut fait, le matin suivant, dans les barattes d'expériences de la fabrique. Pour une moitié de la crème, la température de barattage était de $13^{\circ} \mathrm{C}$. pour l'autre, de $+17^{\circ} \mathrm{C}$. Il y eut quelques points relativement importants pour lesquels les conditions des opérations de la fabrique différaient de celles du laboratoire. D'abord, fait déjà signalé antérieurement, la crème pasteurisée n'était pas refroidie lentement, comme au laboratoire, mais assez rapidement; ensuite, au laboratoire, la température de maturation n'était jamais supérieure à la température la plus basse du barattage, ce qui se produisit à plusieurs reprises, au cours des expériences techniques et dans ce cas la matière grasse de la crème n'était jamais entièrement dans des conditions d'équilibre, se qui aura donné une teneur en matière grasse du babeurre, peut-être un rien plus élevée que celle qui aurait été obtenue si on avait été plus méticuleux. Il y a une troisième différence. Au laboratoire après maturation et avant le barattage, le relèvement de la température se faisait sans que la crème ne fût en contact avec les parois de la baratte qui avaient déjà la température de barattage, tandis qu'au cours des expériences techniques, la crème froide était immédiatement introduite dans la baratte. Comme il a été signalé antérieurement, la baratte était immergée dans de l'eau courante ayant la température du barattage, et il est évident qu'il fallut quelques minutes pour que de la crème, ayant par exemple une température de $\pm 13^{\circ} \mathrm{C}$., fut portée à une température de barattage supérieure de $\pm 4^{\circ} \mathrm{C}$.; une partie du processus de barattage eut donc lieu, à une température trop basse, mais cependant rapidement croissante. Cette dernière différence fut éliminée ultérieurement dans quelques expériences. Il importe encore de signaler que dans un certain nombre de ces expériences, comme dans certaines expériences mentionnées dans l'article précédent, le degré d'acidité de la crème était trop peu élevé; depuis le 5 novembre 1924, les degrés d'acidité étaient plus élevés.

La tableau I donne les résultats obtenus. Dans la plupart des-cas, durant la réfrigération, la crème fut conservée dans deux récipients séparés; il eût été préférable que toute la crème eût séjourné dans un seul récipient durant la maturation, et qu'ensuite on en eût mis

1) Le Lait, 1928, p. 369. 


\section{TABLeaU I}

Influence de la température du barattage sur la teneur en matière grasse du babeurre provenant de crème longtemps rétrigérée à très basse température.

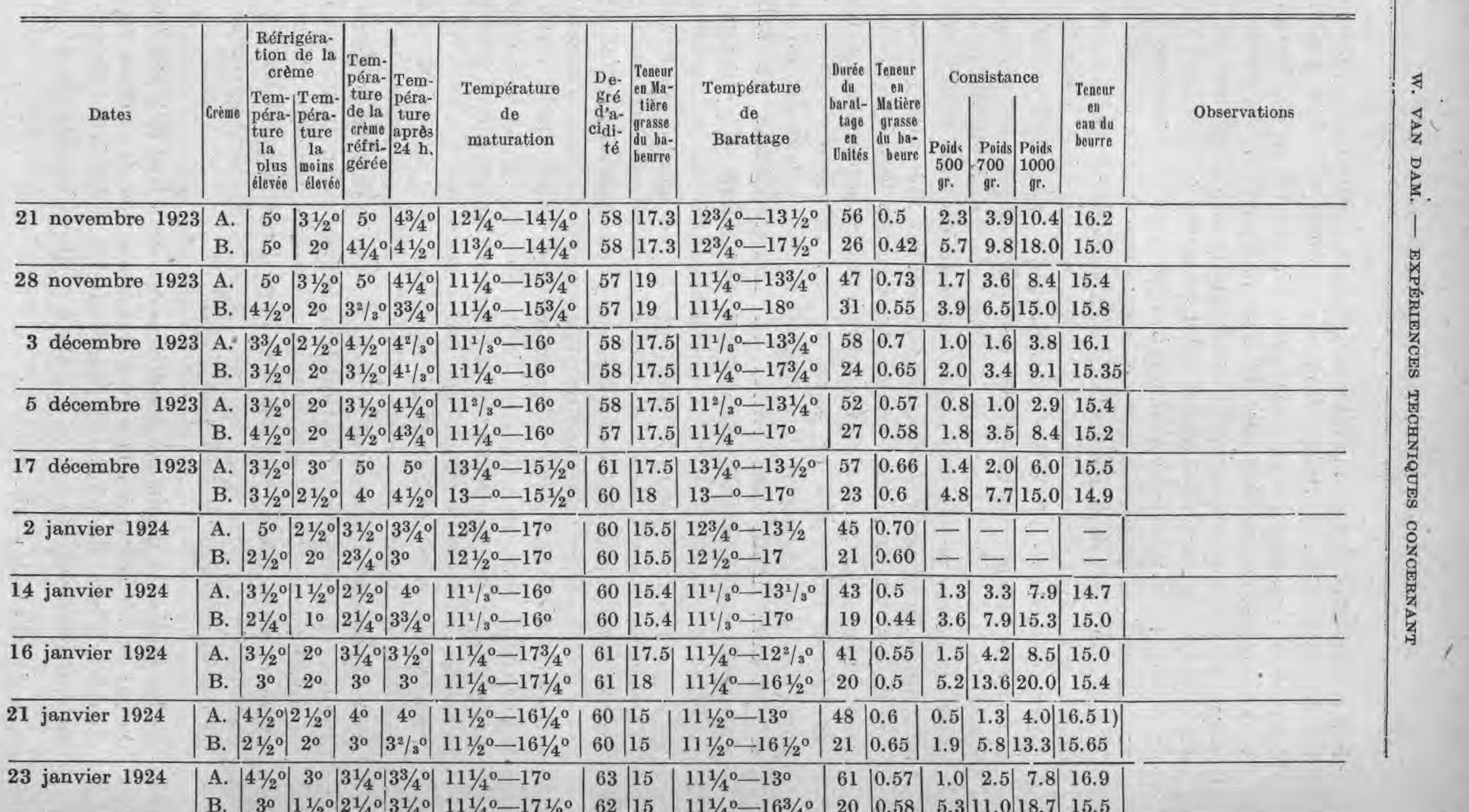




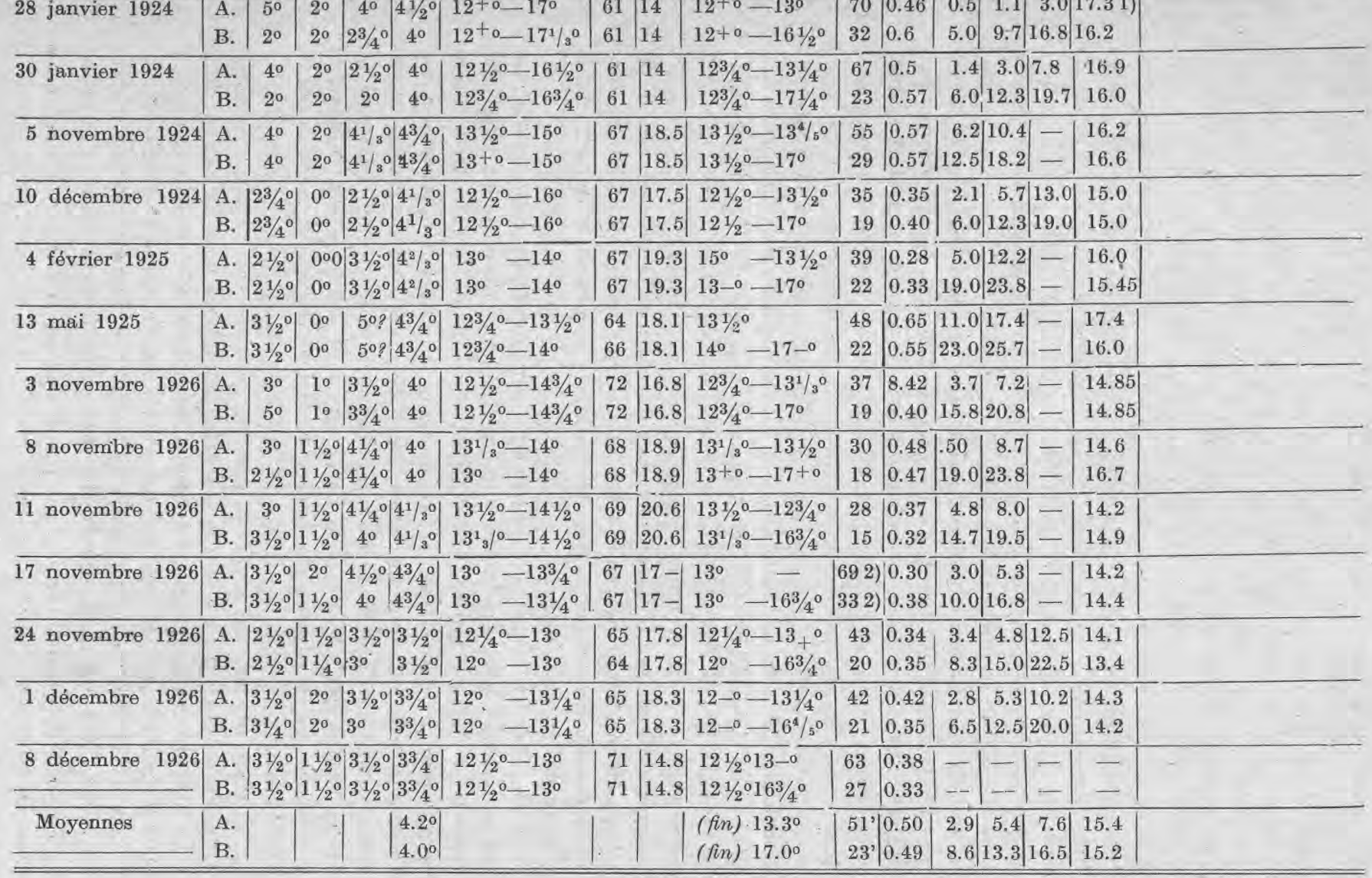

(1) Malaxage en une fois. (2) Mouvement plus lent de la baratte: 41 au lieu de 60 rotations. 
une partie dans une baratte immergée dans de l'eau ayant la température la plus basse, l'autre partie dans la baratte immergée dans l'eau, ayant la température la plus élevée. On peut cependant se rendre compte, par l'examen du tableau I, que les températures de réfrigéra. tion n'ont pas présenté d'écarts notables ; les moyennes de ees températures après +22 heures étaient de $4,2^{\circ} \mathrm{C}$. et $4^{\circ} \mathrm{C}$. La crème $\mathrm{A}$ fut toujours barattée à la température la moins élevée, la crème $\mathrm{B}$ à la température la plus élevée. Les moyennes sont de 13.3 et $17,0^{\circ} \mathrm{C}$.; l'écart qui n'est done pas inférieur à $3^{07} \mathrm{C}$. provoqua une abréviation notable de la durée du barattage de la crème B ; la moyenne pour celui-ci fut de 23 minutes, tandis que pour la crème $\mathrm{A}$, il fut de 51 minutes.

Les teneurs en matière grasse du babeurre ne présentaient que peu d'écart; les moyennes étaient de 0,50 et $0,49 \%$. Tandis que dans 13 sur 23 cas, la teneur en matière grasse du babeurre de la crème barattée à la température la plus basse était la plus élevée, dans 9 cas, elle était la moins élevée. Ces résultats étaient plutôt inattendus, après ce qui fut constaté au laboratoire, où, toujours, la hausse de la température provoqua une hausse de la teneur en matière grasse du babeurre et en présence de ce qui se produit généralement dans la pratique ; mais alors, on a de la crème dont la matière grasse n'est pas dans des conditions d'équilibre. Un second résultat inattendu fut le suivant: la teneur en eau des beurres malaxés n'était pour ainsi dire pas différente, et était en moyenne même un rien moins élevée pour les beurres obtenus par le barattage à la température la plus élevée. Dans 10 sur 19 cas (1), le beurre A avait une teneur en eau plus élevée ; dans 7 cas, une teneur en eau moins élevée que le beurre B et dans 2 cas, la teneur en eau était égale.

J'ai l'intention de faire ultérieurement quelques communications sur la teneur en eau; je mentionne ici quelques données au sujet des teneurs en matière grasse. Les données renseignées au tableau I furent complétées au moyen de celles de quelques autres expériences. Les trois derniers jours d'expérience, quelques litres de la orème furent transportées de la fabrique au laboratoire, pour y être barattés en même temps que se faisait le barattage à la fabrique. La tableau II renseigne sur les durées de barattage et les teneurs en matière grasse constatées.

Comme on pouvait s'y attendre, en présence des résultats obtenus antérieurement avec les petites barattes du laboratoire, la hausse de la température du barattage provoquait une hausse de la teneur en matière grasse du babeurre, tandis que tel n'était pas le cas, à la fabrique.

Des expériences comparatives similaires furent répétées, dans lesquelles il y eut même quatre températures de barattage, et la crème

(1) 2 fols la teneur en eau ne fut pas déterminée, et 2 fois il n'y eut qu'un malaxage pour un des deux lots de beurre. 
TABLEAU II.

\begin{tabular}{|c|c|c|c|c|c|}
\hline \multirow{2}{*}{ Date } & \multirow{2}{*}{ Temp. } & \multicolumn{2}{|c|}{ Durée du barattage } & \multicolumn{2}{|c|}{$\begin{array}{l}\text { Teneur en mat. grasse } \\
\text { du bab eurre }\end{array}$} \\
\hline & & Fabr. & Labor. & Fabr. & Labor. \\
\hline 24 nov. & $\begin{array}{l}13^{\circ} \mathrm{C} . \\
16^{3} / 4^{\circ} \mathrm{C} \text {. }\end{array}$ & $\begin{array}{l}43 \mathrm{~min} \text {. } \\
20 \text { " }\end{array}$ & $\begin{array}{l}37 \mathrm{~min} . \\
17 \text { " }\end{array}$ & $\begin{array}{l}0,34 \% \\
0,35 \%\end{array}$ & $\begin{array}{l}0,25 \% \\
0,45 \%\end{array}$ \\
\hline 1 déc. & $\begin{array}{l}131 / 4^{\circ} \mathrm{C} . \\
163 / 4^{\circ} \mathrm{C} .\end{array}$ & $\begin{array}{l}42 " ~ \\
21 "\end{array}$ & $\begin{array}{l}34 " \\
17 "\end{array}$ & $\begin{array}{l}0,42 \% \\
0,35 \%\end{array}$ & $\begin{array}{l}0,34 \% \\
0,58 \%\end{array}$ \\
\hline 8 déc. & $\begin{array}{l}13^{\circ} \mathrm{C} . \\
163 / 4^{\circ} \mathrm{C} .\end{array}$ & $\begin{array}{l}63 " \\
27 "\end{array}$ & $\begin{array}{l}40 \quad " \\
17 \quad "\end{array}$ & $\begin{array}{l}0,38 \% \\
0,33 \%\end{array}$ & $\begin{array}{l}0,25 \% \\
0,42 \%\end{array}$ \\
\hline
\end{tabular}

ne fut pas seulement réfrigérée à très basse température, mais également refroidie au moyen d'eau de puits NorToN. La tableau III renseigne sur les données obtenues avec de la crème réfrigérée à très basse température.

Tableau III.

\begin{tabular}{|c|c|c|c|c|c|c|c|c|}
\hline \multirow{2}{*}{ Dates } & \multirow{2}{*}{$\underset{\substack{\text { de réfrigération } \\
\text { den }}}{\stackrel{22 \text { heures }}{ }}$} & \multirow{2}{*}{$\begin{array}{c}\text { Température de } \\
\text { maturation }\end{array}$} & \multirow{2}{*}{$\underset{\text { barattage }}{\text { Température }}$ du } & \multicolumn{2}{|c|}{ Duree du barattage } & \multicolumn{2}{|c|}{$\begin{array}{c}\text { Teneur en matière } \\
\text { grasse du babeap re }\end{array}$} & \multirow{2}{*}{\begin{tabular}{|c} 
Teneur \\
en eau \\
du \\
bearre
\end{tabular}} \\
\hline & & & & \begin{tabular}{|c|} 
Pabri- \\
que
\end{tabular} \mid & $\begin{array}{c}\text { Latoria- } \\
\text { toire }\end{array}$ & Falhrique & $\begin{array}{c}\text { Labopa- } \\
\text { toire }\end{array}$ & \\
\hline \multirow{5}{*}{$\begin{array}{l}1926 \\
5 \text { nov. }\end{array}$} & & & & & & & & \\
\hline & $4^{0}-4^{2} / 3^{0}$ & $14 \frac{1}{2^{\circ}-15^{\circ}}$ & $141 / 2^{0}-12^{1 / 3^{0}}$ & $24^{\prime}$ & - & 0.32 & 一 & 13.9 \\
\hline & & & $14 \frac{1}{2^{0}}-14^{\circ}$ & $18^{\prime}$ & 一 & 0.27 & 一 & 13.8 \\
\hline & & & $141 / 2^{0}-151 / 4^{0}$ & $14^{\prime}$ & - & 0.26 & - & 13.8 \\
\hline & & & $\left|14 \frac{1}{2} 0-163 / 4^{\circ}\right|$ & $14^{\prime}$ & 一 & 0.28 & - & 13.5 \\
\hline \multirow[t]{4}{*}{22 nov. } & $33 / 4^{0}-4^{0}$ & $13^{\circ}-13 \frac{1}{1 / 2^{\circ}}$ & $13 \frac{1}{4^{0}-13} 1 / 4^{0}$ & $23^{\prime}$ & - & 0.37 & 0.44 & 14.1 \\
\hline & & & $13 \frac{1}{4^{\circ}-14} 1 / 2^{\circ}$ & $20^{\prime}$ & - & 0.34 & 0.48 & 13.8 \\
\hline & & & $13 \frac{1}{4^{0}-16^{\circ}}$ & $15^{\prime}$ & 一 & 0.32 & 0.53 & 13.7 \\
\hline & & & $13 \frac{1}{4^{0}}-16 \frac{1}{1} 2^{0}$ & 13 & - & 0.39 & 0.57 & 13.5 \\
\hline \multirow[t]{4}{*}{29} & $-33^{3} 4^{0}$ & $-13^{\circ}$ & $12^{\circ}-13^{\circ}$ & $42^{\prime}$ & 35 & 0.22 & 0.29 & 13.9 \\
\hline & & & $12^{\circ}-14^{2} / 3^{\circ}$ & $25^{\prime}$ & $20^{\prime}$ & 0.24 & 0.29 & 13.5 \\
\hline & & & $12^{\circ}-15^{\circ}$ & $26^{\prime}$ & $17^{\prime}$ & 0.30 & 0.33 & 13.5 \\
\hline & & & $\left|12^{\circ}-1634^{\circ}\right|$ & $21^{\prime}$ & $13^{\prime}$ & 0.35 & 0.44 & 13.2 \\
\hline \multirow[t]{4}{*}{6 déc. } & $21 / 2^{0}-3^{0}$ & $121 / 4^{0}-13^{\circ}$ & $121 / 4^{0}-12^{3} /^{0}$ & $54^{\prime}$ & $40^{\prime}$ & 0.35 & 0.24 & - \\
\hline & & & $121 / 4^{\circ}-14 \frac{1}{4^{\circ}}$ & $39^{\prime}$ & $25^{\prime}$ & 0.29 & 0.27 & 一 \\
\hline & & & $12 \frac{1}{4^{\circ}-15} 1 / 2^{\circ}$ & $27^{\prime}$ & $17 \frac{1}{1} \mathbf{2}^{\prime}$ & 0.34 & 0.31 & - \\
\hline & & & 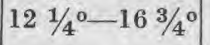 & $21^{\prime}$ & $151 / 2 '$ & 0.34 & 0.39 & - \\
\hline
\end{tabular}

Le 15 novembre, il n'y eut pas encore de barattage comparatif au laboratoire; les données de cette date sont cependant fournies pour montrer que, dans ce cas également, il n'y eut pas hausse de la teneur en matière grasse du babeurre entre les températures de barattage finales, $12{ }^{2} /{ }^{\circ} \mathrm{C}$. et $163 /{ }^{\circ} \mathrm{C}$. Il importe cependant d'observer 
que la température de maturation était trop élevée ici ; elle doit, ainsi qu'il fut signalé antérieurement, être toujours inférieure à la température de barattage la moins élevée, sinon celle-ci s'abaisse durant les premières minutes du barattage, et la crème n'est plus alors dans les conditions d'équilibre, ce qui donne lieu à une teneur plus élevée en matière grasse du babeurre. On peut estimer la teneur $0,32 \%$ tant soit peu élevée, mais on ne peut cependant affirmer qu'il y ait eu dans cette expérience une hausse normale de la teneur en matière grasse. Pour les expériences suivantes, la température de maturation fut suffisamment basse. Dans l'expérience du 29 novembre seule, on put constater une hausse régulière. Dans les trois cas, le babeurre provenant des potites barattes du laboratoire présentait une hausse nette et régulière de la quantité de matière grasse. L'augmentation n'était cependant pas notable, ce qui, ainsi qu'il sera démontré, est probablement dû à la consistance solide de la matière grasse, à la suite de la réfrigération ̀̀. très basse température.

Il importe de signaler la tendance très évidente dans la dernière colonne d'une baisse de la teneur en eau du beurre correspondant à la hausse de la température de barattage. On peut considérer ces données comme concordant avec celles du tableau II de l'article précédent, lesquelles indiquent qu'il y a hausse de la teneur en eau du beurre lorsque $\mathrm{l}_{\mathrm{a}}$ consistance de la matière grasse de la crème augmente.

D'autres expériences furent encore faites avec de la crème simplement refroidie au moyen d'eau provenant de puits NoRTon immédiatement avant l'addition du levain lactique. Le tableau IV rassemble les données obtenues.

Pour la crème pasteurisée, refroidie à $+12^{\circ} \mathrm{C}$. et dont la maturation eut lieu à $+13^{\circ} \mathrm{C}$, le pourcentage de matière grasse du babeurre augmenta progressivement avec la température de barattage. On retrouve done ici ce qui se produit régulièrement dans la pratique et ce qu'on ne constatait pas lorsque la crème était réfrigérée à très basse température et travaillée dans les barattes de la fabrique d'expériences. Le tableau IV montre encore nettement que dans le babeurre provenant des barattes du laboratoire, la hausse de la teneur en matière grasse est plus notable que dans celui provenant des barattes de la fabrique, et si nous comparons ces données avec celles du tableau III, nous

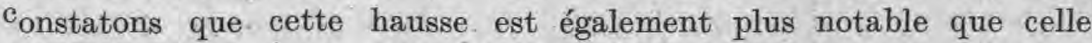
dans le babeurre provenant de crème réfrigérée à très basse température et qụi a été travaillée au laboratoire. Ces données confirment donc la donnée antérieurement obtenue (1) qui permet d'affirmer, qu'en règle générale, pour ce qui concerne la teneur en matière grasse du babeurre, celle-ci est moins influencée par la hausse de la température, lorsque la crème 
Tableau IV.

\begin{tabular}{|c|c|c|c|c|c|c|c|}
\hline \multirow{2}{*}{ Dates } & \multirow{2}{*}{ Réfrigéré à } & \multirow{2}{*}{$\begin{array}{l}\text { Température } \\
\text { de maturation }\end{array}$} & \multirow{2}{*}{$\begin{array}{l}\text { Température } \\
\text { du barattage }\end{array}$} & \multicolumn{2}{|c|}{ Durée du Baraltage } & \multicolumn{2}{|c|}{$\begin{array}{c}\text { Teneur en matière grasse } \\
\text { dn babeurre }\end{array}$} \\
\hline & & & & $\begin{array}{c}\text { Pabri- } \\
\text { que }\end{array}$ & | Laboratoire & Tabrique & Laborateine \\
\hline $\begin{array}{l}1926 \\
13 \text { déc. }\end{array}$ & $12^{\circ}$ & $13^{\circ}-13 \frac{1}{2^{\circ}}$ & $\begin{array}{ll}13^{\circ} & -13 \frac{1}{2^{\circ}} \\
13^{\circ} & -14^{2} 3^{\circ} \\
13^{\circ} & -153^{\circ} \\
13^{\circ} & -17^{\circ}\end{array}$ & $\begin{array}{l}36 \\
30^{\prime} \\
28 \\
23\end{array}$ & $\begin{array}{l}33^{\prime} \\
24^{\prime} \\
22^{\prime} \\
17 \quad 1 / 2\end{array}$ & $\begin{array}{l}0.34 \\
0.38 \\
0.43 \\
0.50\end{array}$ & $\begin{array}{l}0.40 \\
0.50 \\
0.63 \\
0.74\end{array}$ \\
\hline 16 déc. & $12^{\circ}$ & $121 / 2^{\circ}-13 \frac{1}{4^{\circ}}$ & 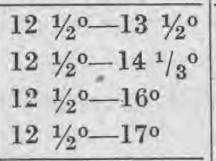 & $\begin{array}{l}43^{\prime} \\
40^{\prime} \\
33^{\prime} \\
28^{\prime} \\
\end{array}$ & $\begin{array}{l}37^{\prime} \\
29^{\prime} \\
211 / 2 \\
20 \\
\end{array}$ & $\begin{array}{l}0.35 \\
0.40 \\
0.47 \\
0.52\end{array}$ & $\begin{array}{l}0.44 \\
0.49 \\
0.68 \\
0.78\end{array}$ \\
\hline 27 déc. & $121 / 2^{\circ}$ & $12^{\circ}-1334^{\circ}$ & 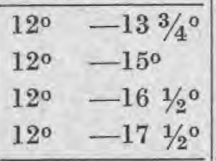 & $\begin{array}{l}55^{\prime} \\
46^{\prime} \\
33^{\prime} \\
29^{\prime}\end{array}$ & $\begin{array}{l}49^{\prime} \\
38^{\prime} \\
26^{\prime} \\
23^{\prime}\end{array}$ & $\begin{array}{l}0.40 \\
0.45^{+} \\
0.47 \\
0.52\end{array}$ & $\begin{array}{l}0.43^{+} \\
0.46 \\
0.55 \\
0.65\end{array}$ \\
\hline $\begin{array}{l}1927 \\
3 \text { jan. }\end{array}$ & $121 / 4^{\circ}$ & $121 / 2^{\circ}-13^{0}$ & 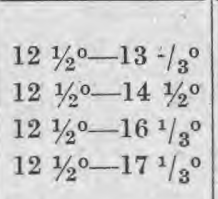 & $\begin{array}{l}48^{\prime} \\
42^{\prime} \\
32^{\prime} \\
29^{\prime}\end{array}$ & $\begin{array}{l}50 \quad 1 / 2 \\
34^{\prime} \\
28^{\prime} \\
21 \quad 1 / 2\end{array}$ & $\begin{array}{l}0.34 \\
0.37 \\
0.45 \\
0.50\end{array}$ & $\begin{array}{l}0.42 \\
0.45 \\
0.63 \\
0.73\end{array}$ \\
\hline 7 jan. & $12 \frac{1}{1 / 4^{\circ}}$ & $121 / 2^{\circ}-13^{\circ}$ & 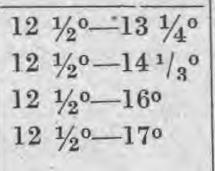 & $\begin{array}{l}57^{\prime} \\
46^{\prime} \\
35^{\prime} \\
28^{\prime}\end{array}$ & $\begin{array}{l}54^{\prime} \\
43^{\prime} \\
31^{\prime} \\
231 / 2\end{array}$ & $\begin{array}{l}0.35 \\
0.39 \\
0.42 \\
0.47\end{array}$ & $\begin{array}{l}0.42 \\
0.48 \\
0.58 \\
0.67\end{array}$ \\
\hline
\end{tabular}

a été longtemps réfrigérée à basse température que lorsque la crème a été moins réfrigérée.

Pour éliminer le dernier point relatif aux différences des conditions du barattage au laboratoire et à la fabrique, nous dirons qu'en raison du fait que la crème, dont la température finale de barattage était de + $17^{\circ} \mathrm{C}$. (un peu plus élevée que celle de l'eau d'immersion des barattes de la fabrique) avait une température de $+13^{\circ} \mathrm{C}$. lors de son entrée dans la baratte, et qu'alors durant les premières minutes la température de barattage était trop basse, quelques expériences furent encore faites à la fabrique, avec de la crème réfrigérée à très basse température qui fut amenée à la température de barattage avant d'être introduite dans la baratte. Comme il fut déjà signalé antérieurement, une telle manipulation n'est pas seulement d'exécution difficile, mais exige plusieurs heures; la matière grasse ne peut venir en contact avec des parois dont la température dépasse la température de barattage. En 
attendant patiemment et en agitant prudemment de temps en temps la crème, on parvient parfois à obtenir le résultat voulu. La crème ainsi traitée après maturation fut barattée ; une moitié (1) à la température de $+13^{\circ} \mathrm{C}$., l'autre à la température de $+17^{\circ} \mathrm{C}$.; la teneur en matière grasse du babeurre fut déterminée et comparée à celle de babeurre provenant de crème identique, barattée au laboratoire, à des températures identiques. De cette façon, il n'y eut de différence que pour ce qui concerne les barattes. Les données sont rassemblées au tableau $\mathrm{V}$.

Tablead V.

\begin{tabular}{|c|c|c|c|c|c|c|c|}
\hline \multirow{2}{*}{ Datez } & \multirow{2}{*}{$\begin{array}{l} \pm 24 \text { heures } \mathrm{de} \\
\text { : Téfrigération } \mathrm{a}\end{array}$} & \multirow{2}{*}{$\mid \begin{array}{c}\text { Températuré de } \\
\text { maturation }\end{array}$} & \multirow{2}{*}{$\begin{array}{l}\text { Tempé- } \\
\text { rature du } \\
\text { barattage }\end{array}$} & \multicolumn{2}{|c|}{ Du rée du barattage } & \multicolumn{2}{|c|}{$\begin{array}{l}\text { Teneur en matière } \\
\text { grasse du babeurre }\end{array}$} \\
\hline & & & & $\begin{array}{c}\text { Fabri- } \\
\text { que }\end{array}$ & $\begin{array}{c}\text { Labora- } \\
\text { toire }\end{array}$ & Fabrique & $\begin{array}{l}\text { Labora- } \\
\text { toire }\end{array}$ \\
\hline \multicolumn{8}{|l|}{1927} \\
\hline \multirow[t]{2}{*}{10 jan. } & $41 / 2^{0}-5$ & $11 \frac{1}{2^{0}}-13^{1 / 30}$ & $13^{1 / 30}$ & $46^{\prime}$ & $38^{\prime}$ & 0.30 & 0.28 \\
\hline & $41 / 2^{\circ}-5^{\circ}$ & $113 / 3^{0}-13^{0}$ & $17^{\circ}$ & $19^{\prime}$ & $20^{\prime}$ & 0.38 & 0.47 \\
\hline \multirow[t]{2}{*}{17 jan. } & $41 / 4^{0}-41 / 2^{0}$ & $1034^{\circ}-13^{\circ}$ & $13^{\circ}$ & $59^{\prime}$ & $47^{\prime}$ & 0.37 & 0.29 \\
\hline & $41 / 4^{0}-41 / 2^{0}$ & $101 / 2^{\circ}-13^{0}$ & $17^{\circ}$ & $22^{\prime}$ & $201 / 2^{\prime}$ & 0.39 & 0.44 \\
\hline \multirow[t]{2}{*}{24 jan. } & $4^{\circ}-4^{3} 4^{\circ}$ & $11^{1} 14^{\circ}-13^{\circ}$ & $13^{\circ}$ & $64^{\prime}$ & $51^{\prime}$ & 0.36 & 0.26 \\
\hline & $4^{\circ}-5^{\circ}$ & $113 / 4^{\circ}-13^{\circ}$ & $17^{\circ}$ & $18^{\prime}$ & $201 / 2$ & 0.40 & 0.47 \\
\hline \multirow[t]{2}{*}{31 jan. } & $4^{\circ} \quad-4 \frac{1}{2^{\circ}}$ & $11^{\circ}-13^{\circ}$ & $13^{\circ}$ & $77^{\prime}$ & $55^{\prime}$ & 0.30 & 0.27 \\
\hline & $4^{\circ}-4^{\circ}$ & $11^{1} / 3^{0}-13^{0}$ & $17^{\circ}$ & $22^{\prime}$ & $23^{\prime}$ & 0.37 & 0.40 \\
\hline
\end{tabular}

La septième colonne verticale montre que dans les quatre expériences de la fabrique, il y avait également baisse de la teneur en matière grasse du babeurre de crème barattée à la fabrique; mais si on compare ces données avec celles du laboratoire, on constate toujours de la divergence au point de vue quantitatif. Que les données du tableau I, pour ce qui concerne la teneur en matière grasse du babeurre aient été influencées par les températures trop basses du début du barattage, au point qu'il n'y avait plus la moindre différence dans la teneur en matière grasse, cela paraît probable si on se rapporte aux données du tableau $V$, mais on ne peut l'affirmer catégoriquement, en présence du nombre restreint d'expériences. Une conclusion définitive ne peut être autorisée que par des expériences plus nombreuses. En examinant toutes les données, on a l'impression nette que l'influence de la hausse de température sur la crème réfrigérée à très basse température est bien moins notable dans les barattes à renversement de la fabrique en ce qui concerne la teneur en matière grasse du babeurre que dans les petites barattes,

(1) A cause de circonstances techniques, la réfrigération et la maturation des deux lots de crème s'est effectuée séparément. 
système Holstein, du laboratoire. L'industrie aura une tendance à attribuer une médiocre valeur aux données obtenues avec ces dernières barattes, et cela à tort, car l'homogénéité de ces données est une garantie de leur valeur; elles indiquent, en outre, que le motif pour lequel il y a défaut de concordance avec les données obtenues à la fabrique, n'est pas sans importance pour la pratique. Comment expliquer que dans les petites barattes, la hausse de la température a une influence plus grande, quand les durées du barattage sont abrégées dans les mêmes proportions pour les deux barattes, et que de ce fait, il faut considérer, l'intensité du mouvement communiqué à la crème comme à peu près égale? Il est possible que cette intensité soit à peu près égale et qu'il y ait formation d'écume, à peu près égale, dansles deux barattes, mais que dans les petites barattes système Holstein, dans lesquelles il y a des agitateurs à palettes, une partie de la crème est constamment projetée contre les bords en arête des palettes, d'où morcellement des conglomérats de matière grasse déjà formés et, de ce fait, augmentation de la teneur en matière grasse du babeurre (1). Dans les barattes à renversement, cette destruction qu'on pourrait qualifier «locale» des conglomérats formés (selon RAHN) dans l'écume n'a pas lieu ou elle ne se fait que dans de moindres proportions. Continuant dans cet ordre d'idées, on pourrait supposer que la persistance de la matière grasse dans le babeurre pourrait bien être attribuée, non pas au fait que des globules de matière grasse échappent à la conglomération, mais à la circonstance que des conglomérats déjà formés sont morcelés. Dans ce cas, le résultat du barattage serait en premier lieu une résultante de deux forces simultanément actives : une force conglomérante et une force désintégrante. Cette hypothèse est plus ou moins étayée par une expérience que je fis en collaboration avec MHe HoLwERDA, dans laquelle la crème fut barattée dans des conditions telles que les circonstances pouvant favoriser le défaut d'agglomération des globules de matière grasse étaient augmentées, tandis que celles favorisant le morcellement des conglomérats formés étaient diminuées. La teneur en matière grasse du babeurre fut très appréciablement réduite. Ces expériences seront exposées dans un article ultérieur. En terminant, j'attire encore l'attention sur le fait que tout ce qui précède se rapporte à du beurre préparé de crème de lait provenant de vaches en stabulation, que jusqu'ici il ne fut pas encore fait d'expériences avec de la crème de lait provenant de vaches au régime d'été.

(Trad. R: N. Göransson).

\footnotetext{
1 RAHN a déjà attiré l'attention sur la possibilité du morcellement de la matière grasse, déjà solidifiée; Forschungen auf den Gebiet der Milchwirschaft und Molkereiwesens. II. 76. (1922).
} 